\title{
The role of the liver in the migration of parasites of global significance
}

\author{
Gwendoline Deslyper ${ }^{1 *}$, Derek G. Doherty ${ }^{2}$, James C. Carolan ${ }^{3}$ and Celia V. Holland ${ }^{1}$
}

\begin{abstract}
Many parasites migrate through different tissues during their life-cycle, possibly with the aim to enhance their fitness. This is true for species of three parasite genera of global importance, Ascaris, Schistosoma and Plasmodium, which cause significant global morbidity and mortality. Interestingly, these parasites all incorporate the liver in their lifecycle. The liver has a special immune status being able to preferentially induce tolerance over immunity. This function may be exploited by parasites to evade host immunity, with Plasmodium spp. in particular using this organ for its multiplication. However, hepatic larval attrition occurs in both ascariasis and schistosomiasis. A better understanding of the molecular mechanisms involved in hepatic infection could be useful in developing novel vaccines and therapies for these parasites.
\end{abstract}

Keywords: Liver, Migration, Immunotolerance, Ascaris, Schistosoma, Plasmodium

\section{Background}

The life-cycle of many parasites in their final host often involves migration from one tissue to another. The reasons for this are unclear; however, tissue migration has been linked to increased body size and maturation, which leads to improved parasite survival [1]. A recent study [2] suggested that tissue migration enables the parasite to avoid eliciting an immune response, which is often raised at mucosal surfaces. If evading the host's immune response is in fact a key purposes of this migration, the potential role of the liver becomes clear. The liver is an immunotolerant organ and therefore an ideal place for parasites to hide from the immune system. We will use human parasites of three genera, Ascaris, Schistosoma and Plasmodium, to illustrate the essential role of the liver in the life-cycle of these parasites. We will demonstrate that the liver is a crucial step in their life-cycle, a point at which infection appears to go unnoticed, but a potential bottleneck where vaccination/treatment could be most effective.

\footnotetext{
*Correspondence: deslypeg@tcd.ie

${ }^{1}$ Department of Zoology, School of Natural Sciences, Trinity College

Dublin, Dublin 2, Ireland

Full list of author information is available at the end of the article
}

\section{Immunotolerance in the liver}

The special immune status of the liver was first identified in transplantation experiments in pigs, where allogeneic liver transplants were not rejected as was the case with other organs [3, 4]. In humans some tolerance is observed whereby transplanted livers recover spontaneously after a rejection reaction [4] and some liver allograft recipients can even be completely withdrawn from immunosuppression [5].

Hepatic immunotolerance occurs through a combination of unique anatomical and histological features of the liver. Most of the blood that enters the liver comes directly from the portal system, making it the first organ to be exposed to gut-derived molecules including harmless bacterial products and nutrients [6]. The smallest unit of the liver, the hexagonal lobule, consists of a small layer of hepatocytes around a central vein. The capillary bed of the liver, the sinusoids, does not form tight junctions, instead it forms fenestrations that are known as sieve plates [3]. The perisinusoidal space, also called the space of Disse, replaces the basement membrane to separate the liver sinusoidal endothelial cells (LSECs) from the hepatocytes [3].

The liver sinusoids are home to multiple populations of resident immune cells. These include myeloid leukocytes and liver parenchymal cells that express receptors that 
sense pathogens, and myeloid and lymphoid cells capable of phagocytosis and cytotoxicity [7]. Central to the tolerogenic nature of the liver, the sinusoids contain multiple populations of antigen-presenting cells (APC) which present antigenic peptides bound to major histocompatibility complex (MHC) molecules to $\mathrm{T}$ lymphocytes of the adaptive immune system [7-9]. Hepatic APCs are capable of activating $\mathrm{T}$ cells in vitro, inducing cytotoxicity and inflammatory cytokine secretion [7]. However, in the environment of the liver, hepatic APCs are more likely to inactivate $\mathrm{T}$ cells or induce their maturation into regulatory $\mathrm{T}$ (Treg) cells that suppress immune responses [3].

Antigen presentation to $\mathrm{T}$ cells is typically mediated by dendritic cells (DC) [10]. DCs express pathogen receptors that enable them to recognise components of microorganisms that are not found in mammalian systems. They also express costimulatory and/or coinhibitory receptors and release cytokines that determine the nature of $\mathrm{T}$ cell activation or $\mathrm{T}$ cell tolerance. Antigen presentation by hepatic $\mathrm{DC}$ generally results in $\mathrm{T}$ cell inactivation by anergy or exhaustion. These tolerogenic DC can also drive the differentiation of naïve $\mathrm{T}$ cells into Treg cells, which release immunosuppressive cytokines and suppress the activities of other immune cells in an antigenspecific manner [11-13].

Macrophages, known as Kupffer cells, are also abundant in the liver sinusoids. Similar to other macrophages, two subsets of Kupffer cells (KC), defined by their phagocytic and cytokine-producing properties, have been described [14]. 'Inflammatory' or M1 macrophages secrete high levels of the proinflammatory cytokine IL-12 and low levels of the regulatory cytokine IL-10, whereas 'alternatively-activated' or 'repair' M2 macrophages, produce high levels of IL-10, TGF- $\beta$ and low levels of IL-12 [15]. Upon pathogen receptor ligation, KCs most frequently act as M2 macrophages [16] and antigenpresentation by these cells is frequently associated with expression of inhibitory ligands and cytokines and the induction of Treg cells $[17,18]$.

LSECs also express MHC and costimulatory molecules and are capable of presenting antigen to $\mathrm{CD} 8^{+} \mathrm{T}$ cells leading to tolerance [19-21] and to $\mathrm{CD}^{+} \mathrm{T}$ cells leading to their differentiation into Treg cells [22]. Hepatic stellate cells (HSC), also called Ito cells, can also present antigen to $\mathrm{T}$ cells [23], but again, antigen presentation by this APC preferentially promotes $\mathrm{T}$ cell tolerance $[24,25]$. HSC can also promote the differentiation of monocytes into myeloid-derived suppressor cells (MDSC), which have potent $\mathrm{T}$ cell inhibitory activities [26]. Hepatocytes also express pathogen receptors, MHC and costimulatory molecules, although it is not clear these cells can present antigens to $\mathrm{T}$ cells leading to their activation $[27,28]$.

\section{Three parasite genera of global importance: Ascaris, Schistosoma and Plasmodium}

Species of the three parasite genera discussed in the present review all use different ways to enter their final hosts, oral ingestion of eggs (Ascaris spp. [29]), skin penetration by free-swimming cercariae (Schistosoma spp. [30]), and injection into the blood stream via mosquito bites (Plasmodium spp. [31]) (see Fig. 1). Despite entering different tissues, the parasites migrate to the liver rather quickly.

After egg-hatching in the gut, Ascaris spp. larvae are transported to the liver via the portal vein. Subsequently the larvae migrate to the lungs, where they are coughed up and swallowed thereby re-entering the gut [32]. The complexity of this life-cycle, in addition to the fact that both the start and end organ of the life-cycle are one and the same, the gut, indicates the importance of tissue migration, potentially related to increased fitness of the parasite.

Schistosoma spp. are slightly different, as the parasites migrate to the a different organ, the lungs first, before entering the liver and ultimately reaching the mesenteric vessels [30]. This is therefore the opposite path that Ascaris spp. larvae take. Although Schistosoma spp. reach the lungs first and the liver second, we will show that the role of the liver in the migratory path is still very important in the larval development. In particular, in nonimmune animals, the liver appears to play an important role in parasite attrition.

Plasmodium sporozoites are injected into the skin by an infected mosquito and migrate to the liver via the bloodstream [33] where the parasites mature into merozoites and multiply. Subsequently the merozoites are released in great numbers into the bloodstream and infect erythrocytes where they mature into trophozoites, schizonts which release merozoites during asexual multiplication [33]. Ultimately trophozoites will mature into male and female gametocytes that can be picked up by a mosquito

(See figure on next page.)

Fig. 1 The migratory path of Ascaris, Plasmodium and Schistosoma. Ascaris eggs are ingested orally and after hatching, larvae penetrate the gut wall. The larvae subsequently move to the liver and the lungs from where they are coughed up and swallowed thereafter establishing as adult worms in the gut. Plasmodium sporozoites are injected into the skin; from there they migrate to the liver where they multiply into merozoites. Merozoites leave the liver and infect erythrocytes where they eventually mature into gametocytes to continue the life-cycle. Schistosome cercariae are released into water from infected snails. Cercariae penetrate the skin and then migrate to the lungs and then the liver. Ultimately, these parasites establish themselves as adult worms in the mesenteric veins 


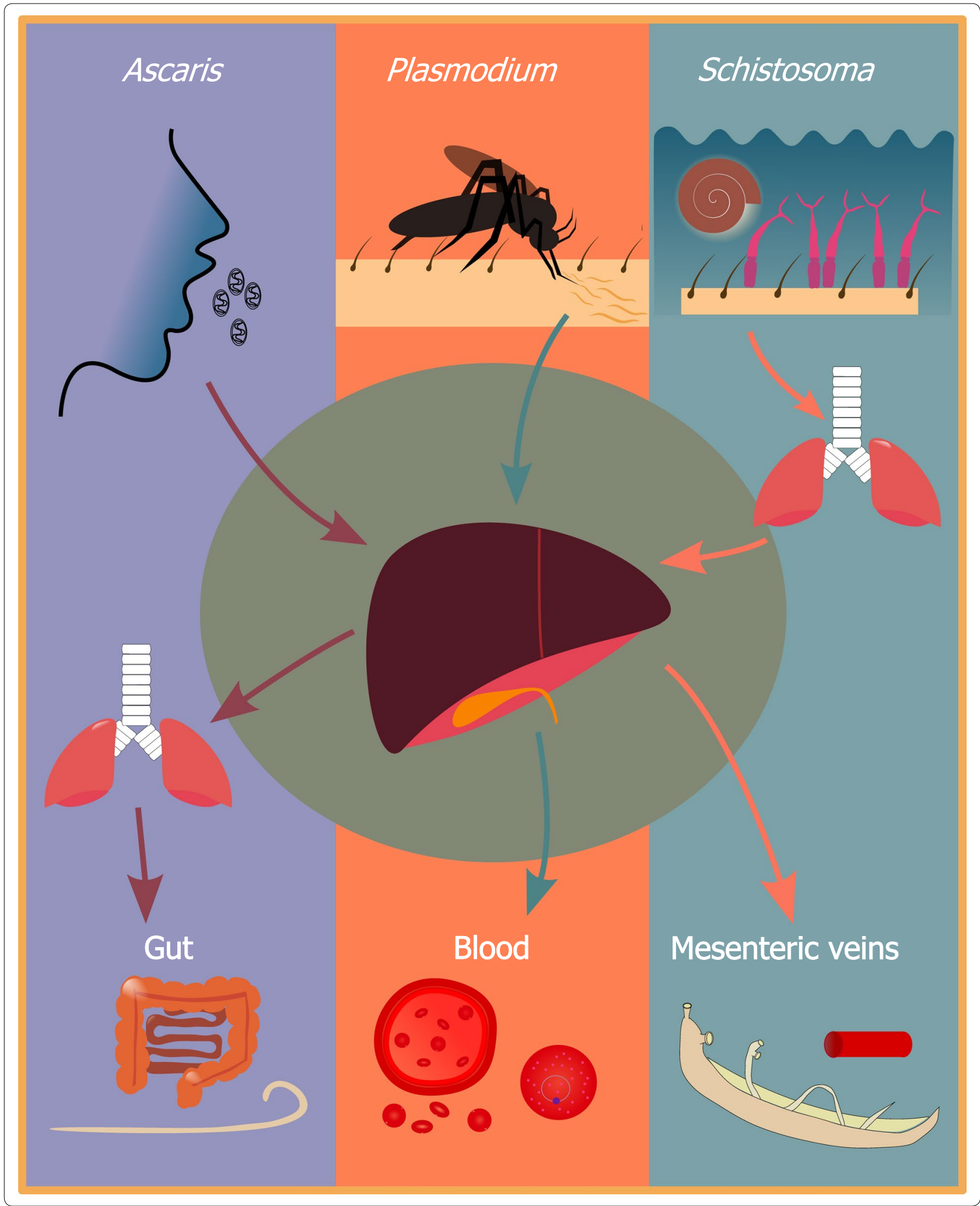


bite. The liver is therefore used by the parasite as a 'safe haven' for the parasites to evade the hosts' immune system. It is also the place where hypnozoites from $P$. vivax can remain unnoticed for years before restarting their life-cycle and causing malaria pathology.

\section{Ascaris: self-cure and differential burden}

Ascaris lumbricoides is a soil-transmitted helminth which infects 800 million people worldwide [34]. The eggs have a thick shell, making them highly resilient to various environmental factors such as temperature and desiccation [35]. Infections usually peak in children between 5-15 years-old, who can experience symptoms ranging from growth retardation to diminished cognitive development $[35,36]$.

Most infected individuals carry light worm burdens but a relatively small proportion harbour heavy infections, a distribution described as aggregated [37]. Predisposition to infection has also been observed in ascariasis, a phenomenon whereby individuals are prone to a particular intensity of infection and regain similar worm burdens after treatment [38]. Although the exact mechanism of predisposition is unknown, it has been found to involve various factors, such as host genetics and adaptive immunity [39].

While the liver stage appears to be clinically silent, the presence of macroscopical white spots, areas of inflammation formed around the larvae due to injury during migration, in the liver of both humans infected with $A$. lumbricoides [40] and pigs infected with the porcine ascarid A. suum [41] is a clear indication that an immune response is generated. When treating experimentally infected pigs with anthelmintics during the liver stage (day 2, 3 and 4 post-infection, p.i.) of $A$. suum infection, an increase of $22 \%$ in their feed conversion rates, was found when compared to treating the pigs at the lung stage of infection (day 6, 7 or 8 p.i. [42]). As for humans, a prospective study of 510 Indian patients with liver abscesses during a 10-year period identified A. lumbricoides as the causative agent in $14.5 \%$ of the cases [40]. Similarly, a study in South African children demonstrated that $A$. lumbricoides was the causative agent of liver abscesses in $2 \%$ of the cases [43].

\section{Self-cure in pigs}

Self-cure is a phenomenon observed in some pigs, where pigs receiving an oral trickle infection with $A$. suum eggs do not develop intestinal worms. The potential role of the liver in this phenomenon has received considerable attention.

In order to assess the role of the liver, an experiment was performed which bypassed the liver by infecting pigs with L3 stage (the lung stage) larvae through intravenous injection [44]. At day 21 p.i., a time at which self-cure usually has occurred, pigs did not show the typically observed decreased larval burden. This led the authors to believe that the liver played a crucial role in self-cure. However, the lack of a control group of orally infected pigs makes it hard to confirm this hypothesis. Another study approached the question of the role of the liver in self-cure by orally infecting pigs with lung-stage larvae [45]. The authors found first that self-cure still occurred, and secondly, that it happened at the same rate as their controls, i.e. pigs orally infected with embryonated eggs. A weep and sweep response has been attributed to this observation, whereby increased mucus secretion and gut movement eliminates the larvae before they can penetrate the gut wall [46]. Additionally, intestinal eosinophils and $\mathrm{T}$ cells were found to potentially play an important role. The mechanisms behind self-cure appear to be diverse and are not yet fully understood.

\section{A mouse model for hepatic resistance}

In order to study the liver stage of ascariasis, an appropriate animal model is necessary. Building on earlier work [47], a mouse model has been developed specifically to study the predisposition phenomenon by using two inbred mouse strains which mimic the extremes of predisposition to light and heavy infection [36, 48, 49]. CBA/ $\mathrm{Ca}$ and $\mathrm{C} 57 \mathrm{BL} / 6 \mathrm{~J}$ were found to have a substantial and consistent difference in lung larval burden, with the former as the relatively resistant strain and the latter as the relatively susceptible strain.

This mouse model was subsequently used to investigate when, during migration, this difference in larval burden occurs [50]. Mice of both strains were infected with the same number of $A$. suum eggs. At regular intervals from 6 hours p.i. until 8 days p.i., mice were sacrificed and their organs (gut, liver, lungs) removed. The larvae were retrieved from those organs, using the modified Baermann method. The larvae were subsequently enumerated and counted. This allowed for detailed tracking of larval migration throughout the various organs. Using this mouse model, similar larval burdens were found in the liver of both strains [50]; however, once the larvae reached the lungs a significant difference in larval burden was observed, with the relatively resistant strain having a lower burden than the relatively susceptible strain. Another study found similar results, with no difference in total larval burden in the liver between the two strains [49]. The first larvae appear in the liver at 6 hours p.i. with a peak appearing between days 3-6, at this stage the larval burden is similar in both strains [50]. The larvae subsequently migrate to the lungs, with the first larvae appearing as early as day 1 p.i.; however, the majority of the larvae arrive around day 6 p.i. At this 
point it becomes clear that one strain is relatively susceptible (C57BL/6J) and another relatively resistant (CBA/ $\mathrm{Ca})$, as there is a statistically significant difference in lung larval burdens between the two strains. Interestingly, the authors pointed out that there is a steady increase in mean larval burden in the liver between days 2 and 5 p.i., long after the larvae should have penetrated the gut wall and made their way to the liver. The authors speculated that "larvae were arriving from other locations and perhaps had become temporarily lost or trapped in other host tissues", indicating a strong instinct for the larvae to make their way to the liver and highlighting the importance of this organ during migration.

The question then remains, which organ contributes most to this difference in infection rate, is it the departing organ, the liver, or the organ where the larvae arrive, the lungs. In order to explore this question in more detail, an investigation of the presence of various leukocytes in the bronchoalveolar fluids (BAL) in both strains of mice, infected with A. suum [50]. This study showed that an increase in leukocytes occurred at days $8-9$ p.i., so only after the difference in larval burden had already occurred. Additionally, it was found that the increase in leukocytes in BAL was higher in the susceptible strain than in the resistant strain, indicating that this increase did not contribute to the difference in larval burden between the two mouse strains but reflected the observed larval burden. This was confirmed in the same study by examining the lung tissue, in which was observed a greater inflammatory response in the susceptible strain. Taken together, these data indicate the importance of the liver stage and that it plays a key role in the difference in infection rate between these two mouse strains.

Shotgun mass spectrometry was used to explore the liver proteome in the above mentioned mouse model at day 4 p.i. [51]. We observed a difference in abundance of mitochondrial proteins involved in oxidative phosphorylation. The relatively resistant strain $(\mathrm{CBA} / \mathrm{Ca})$ had, both intrinsically and under infection, higher levels of this protein group than the relatively susceptible strain $(\mathrm{C} 57 \mathrm{BL} / 6 \mathrm{~J})$. This led us to believe that a potential intrinsic difference in reactive oxygen species (ROS) in the liver gives the relatively resistant strain an advantage in contending with the parasite.

A subsequent study investigated the difference in liver proteome at day 7 p.i. This study confirmed the earlier findings of day 4 p.i., demonstrating that the relatively resistant strain had a higher abundance of proteins involved in oxidative phosphorylation. However, the later day experiment revealed an important difference in the immune response to A. suum in the liver, with the relatively susceptible strain showing a higher abundance of proteins involved in complement inactivation compared to the relatively resistant strain which had a higher abundance of proteins involved in complement activation [52]. These two studies demonstrate the importance of delineating host responses to helminth infection at different time points post-infection.

During a reinfection experiment [53], BALB/c mice were infected with $A$. suum, the authors found no difference in the liver larval burden when comparing mice that were infected once or reinfected. However, a lower larval burden was observed in the lungs in the reinfected mice, and more importantly, lesions caused by hepatocyte necrosis and infiltration of eosinophils and neutrophils were more pronounced in the reinfected group. These results indicate that the observed, more pronounced hepatic immune response in the reinfected group results in a decrease in lung larval burden.

To conclude, the presence of white spots indicates the presence of a strong immunological response in the liver. The liver stage becomes particularly important when investigating predisposition in pigs, mice and humans. Novel therapies could therefore focus on the liver in order to stop larval migration in its tracks, prevent increased damage due to larval migration and ultimately inhibit the development of adult worms which have significant impact on the nutritional and developmental status of the host $[51,54]$.

\section{The liver as a site for attrition in Schistosoma infection}

Species of Schistosoma infect over 250 million people worldwide [30]. Reinfections are common in endemic regions, with children often having suffered their first infection by the age of two, followed by a steady increase in worm burden with every new infection [55]. After these early infections, the worm burden decreases with increased exposure to the parasite that is thought to increase immunity, combined with the death of older worms [30].

Schistosoma spp. interact with the liver during two distinct parts of their life-cycle. First, for all human Schistosoma species, the immature worms pass through the liver's vasculature after lung migration. Secondly, after the migratory path has been completed and the adult worms are located in the mesenteric vessels, some excreted eggs however do not end up in the faeces but instead travel to the liver via the hepatic vessel, this is true for all human Schistosoma species except S. haematobium. Schistosoma haematobium is the only species that does not cause liver pathology, as the adult worms are located in the venus plexus of the bladder [56]. In order to break down the eggs, granulomas are formed around them, causing hepatic fibrosis, which is the main source of mortality and morbidity in chronic 
schistosomiasis [57]. Liver-associated disease in schistosomiasis is more common in S. japonicum and $S$. mansoni infections [58]. The liver is also profoundly impacted due to the longevity of the adult worms with an average life span of 3-10 years [59]. In essence, the most damaging symptoms associated with Schistosoma spp. infection, hepatic granuloma formation does not occur until after larval migration is completed and can be considered an unwanted side effect.

\section{The liver as a site for maturation, pairing and sexual development}

The liver stage is a crucial phase in the life-cycle of schistosomes, it is here that they can increase their biomass and develop into mature life stages $[60,61]$. These important life-cycle changes do not occur at any other stage, in fact, schistosomulae of $S$. mansoni which were trapped in the pulmonary vasculature were not able to reach maturity $[60,62]$. Parasites that reached the liver, however, showed exponential growth, thus demonstrating that the liver's vasculature alone can provide the parasites with an adequate environment to reach maturity. Additionally, it was found that culturing $S$. mansoni schistosomulae in the presence of human portal serum showed a significant increase in cell proliferation when compared to schistosomula cultured in the presence of human peripheral serum [63,64], again confirming that the liver vasculature provides the optimal environment for these parasites. However, so far it is not clear which components contribute, or might be essential for these processes to occur.

Additionally, it is at the liver stage that male and female parasites pair, which allows the females to reach sexual maturity; females cannot reach sexual maturity without the presence of males [65]. The paired schistosomes subsequently migrate against the blood flow to the mesenteric veins [66]. The fact that these parasites need to manoeuvre against the blood flow, a process that female parasites cannot do by themselves [66] highlights again the importance of the liver stage for this parasite.

\section{Attrition: when, where and how?}

The site of attrition has been a topic of contention for decades, it was studied intensively in the 1980s thanks to the development of the autoradiographic tracking [67] which greatly improved the sensitivity of these experiments. Using this technique, it was found that between $86 \%$ and $90 \%$ of the skin-penetrant cercariae in naïve mice (i.e. mice that received a single round of infection) had migrated to the lungs, indicating that the skin was not the site of attrition [68, 69]. Further investigation of the migration of $S$. mansoni, using autoradiographic tracking in C57BL/6 mice showed a peak in the lungs at day 8 and in the systemic organs at day 12 [70]. All this indicates attrition occurring between the pulmonary and hepatic life stages of the parasite [68]. This raises the question as to why these parasites move to the liver next - perhaps the liver is a safe haven - a place where the parasites are no longer under attack and can reach sexual maturity safely hidden from the immune system.

As for immunized animals, which were immunized through either gamma-irradiated cercariae or establishing a chronic infection, the attrition is largely similar to that of naïve animals, except that everything appears to go more slowly, i.e. migration from the skin to the lungs takes longer and peak burden in the lungs was at a later time point. The big difference with naïve animals is the lower number of parasites reaching the systemic and splanchnic organs [70-73].

The location of attrition varies between Schistosoma species. For S. mansoni and S. haematobium attrition mainly occurs in the lungs $[74,75]$. However, five times more $S$. mansoni larvae reached the liver than is the case for S. haematobium. This observed greater larval death in S. haematobium infection can be partially attributed to the fact that mice are non-permissive hosts for this parasite [76]. Attrition in S. japonicum was found to occur in both the lungs and the liver [77].

Regardless of whether the lungs or the liver are the main site of larval removal, it is still crucial to investigate the role of the liver in the life-cycle, which could also be a potential target for vaccination. Early removal of the parasite, before it reaches sexual maturity and therefore egg-laying, would reduce granuloma formation.

\section{Multiplication of Plasmodium in the liver}

The protist parasites Plasmodium spp. are the causative agents of malaria in humans, resulting in an estimated 429,000 deaths in 2015 [78]. There are five species that infect humans: $P$. falciparum, $P$. ovale, $P$. vivax, $P$. malariae and $P$. knowlesi. Of these, $P$. falciparum infection is the most common and pathogenic, causing $99 \%$ of malaria associated deaths [78].

Malaria-associated pathology is often divided into uncomplicated and severe P. falciparum malaria. Uncomplicated malaria results in general malaise [31]. Severe falciparum malaria is caused by $P$. falciparum sequestering in the small and medium-sized blood vessels of different organs particularly the brain [31]. Pathology in humans is therefore mainly associated with the blood stages of the parasite and complications thereof such as cerebral malaria [31]. The lack of symptoms and the brief duration of the liver stage, make it a particularly difficult stage to study. However, a recent study using malarianaïve human volunteers who were infected with $P$. falciparum through infective bites observed an increase in 
total leukocyte, lymphocyte and monocyte count during the liver stage followed by a decrease in the aforementioned counts when the blood stage is initiated [79].

The liver is the only organ necessary for Plasmodium spp. maturation. At this stage a low number of sporozoites proliferate into a large numbers of merozoites, ready to invade erythrocytes and start the process of the wellknown cyclic fever bouts. Due to this bottleneck, the liver stage forms an ideal target for vaccination purposes. However, before a vaccine can be developed, an in-depth understanding of this stage is necessary. The National Institute of Allergy and Infectious Diseases recently identified 'greater understanding of parasite liver-stage biology and development' as a key challenge in malaria vaccine development [80].

\section{The path to the liver}

After being deposited in the skin from a mosquito bite, sporozoites migrate to the liver via the blood. To enter the liver, the sporozoites must cross the fenestrated endothelial layer; however, the fenestrations are too small for the sporozoites to pass through, thus passage must go through the sinusoidal cells [81]. Circumsporozoite protein (CSP) and thrombospondin-related anonymous protein (TRAP), expressed by sporozoites, binds human heparin sulphate proteoglycans (HSPGs), the signal for sporozoites to leave the blood stream [82, 83]. Whether the sporozoites invade the liver through $\mathrm{KC}$ invasion or not is still under debate, with some studies suggesting a necessary step through KCs [84, 85], whereas others indicating that hepatocytes can be invaded directly [86]. Some studies suggest that sporozoites need to travel through KCs, whereas others indicating that hepatocytes can be invaded directly. Sporozoites have been shown to directly infect hepatocytes in vitro and develop into merozoites; however, due to the cellular structure of the liver it has been suggested that in vivo the sporozoites must pass through KCs [84]. Frevert et al. [85] described the migration of the sporozoites with an abrupt speed change, at which point they glide along LSECs, followed by a long pause for the sporozoites to enter the KCs, which the authors attribute to formation of nonfusogenic vacuole formation and a relatively slow passage through the KCs. CSP from sporozoites has been shown to elevate cAMP levels in KCs, thereby inhibiting the cells from producing a respiratory burst and thus protecting the sporozoites [87]. Additionally, sporozoite microneme protein essential for cell traversal 2 (SPECT2) deficient sporozoites were not able to infect the liver in vivo [88]. These data suggest the need of KCs in hepatocyte infection.

However, a recent study provided evidence for the alternate hypothesis [86]. The authors, building on earlier work [89], identified that $17 \%$ of sporozoite cell traversals exclusively involve endothelial cells [86]. They also identified that $15 \%$ of crossing events were independent of cell traversal and KCs, which could be the sporozoite moving between two endothelial cells or between an endothelial cell and a KCs. Furthermore, the authors found that sporozoites KCs traversal is associated with cell death of those KCs.

Regardless of this, sporozoites will eventually migrate through several hepatocytes, for yet unknown reasons, using a transient vacuole to ensure passage [85]. Three theories exist as to why the Plasmodium parasites travel through several hepatocytes before forming the PV and ultimately differentiating into merozoites. The first theory argues several rounds of migration through hepatocytes might be a protective mechanism by the parasite to ensure that formation of the PV and subsequent merozoites formation only occurs in the liver and not in the other tissues (skin, etc.) [89]. A second theory builds on the observation that hepatocyte growth factor is released when the parasite migrates through hepatocytes this induces neighbouring hepatocytes to be more susceptible to infection [90]. Thirdly, it has been suggested that upon detection of high sulphated HSPGs, the sporozoites turn off their traversal machinery and activate the invasion machinery; however, this has been shown to take between 30-60 min in Plasmodium yoelii, which would explain the invasion of multiple hepatocytes [91, 92]. When reaching their final hepatocyte, a parasitophorous vacuole $(\mathrm{PV})$ is formed and the sporozoites can differentiate into merozoites [85].

The merozoites will then need to pass the space of Disse, at which stage they are vulnerable to KC phagocytosis [93]. To avoid this, the infected hepatocytes release merozoite-filled vesicles, which are derived from the plasma membrane of hepatocytes, into the liver sinusoids $[93,94]$. The phosphatidylserine switch is prevented in these merozoites-filled vesicles so as not to alert the $\mathrm{KC}$ and other immune cells, granting an escape route for the parasite [93].

\section{Hypnozoites}

Hypnozoites have only been observed in P. vivax [95], although a handful of cases of relapsing $P$. ovale have been seen as well, but without confirmation of hypnozoites [96]. This life stage arguably exploits the liver's special immune status to the fullest. By going into a dormant state in the liver, for weeks or months, these parasites can hibernate and resume infection [97]. No study so far have been able to identify the triggers for hypnozoites reactivation, nor its specific relationship to the liver.

The importance of these hypnozoites, which can remain dormant for 6-9 months, cannot be overstated 
if the ultimate goal is malaria elimination $[98,99]$. Up to $80 \%$ of all blood stage $P$. vivax infections are attributed to relapses; however, not all relapses can be attributed to hypnozoites and other mechanisms are thought to be at play [100-102]. Investigating these hypnozoites more in depth could give a better insight in to why Plasmodium spp. incorporate the liver in the life-cycle and how they exploit hepatic immune tolerance.

\section{Hepatic immunity}

Naturally acquired immunity is probably not achieved at the liver stage, rather it is more likely an antibody-mediated response to the blood stage [33]. However, the liver stage offers a great opportunity to activate the immune system and eliminate the relatively few sporozoites before they multiply greatly in numbers and spread all over the body via the blood.

Innate recognition of Plasmodium RNA by melanoma differentiation-associated gene 5 protein (MDA5) and mitochondrial antiviral signalling (MAVS) pathway in infected hepatocytes induces activation of the transcription factors interferon-regulatory factors-3 (Irf3) and IRF7 [103]. Additionally, a recent in vivo study showed that protection against clinical malaria in children is associated with $\mathrm{C} 1 \mathrm{q}-$-fixing antibodies against CSP in $P$. falciparum sporozoites [104]. These antibodies inhibited hepatocyte cell traversal and ultimately induced sporozoite death.

$\mathrm{CD}^{+} \mathrm{T}$ cells play an important role in immunity to malaria, with $\mathrm{CD} 8^{+} \mathrm{T}$-cell depleted mice being unable to develop immunity [105]. The efficacy of parasite inhibition is therefore dependent on the availability of effector $\mathrm{CD}^{+} \mathrm{T}$ cells [106]. Some $\mathrm{CD} 8^{+} \mathrm{T}$ cells get primed by dendritic cells in the skin draining lymph nodes before moving to the liver where they eliminate antigen presenting hepatocytes [107]. No evidence exists that hepatocytes can successfully present antigen and activate naive $\mathrm{CD}^{+} \mathrm{T}$ cells, however, hepatocytes can present a CSP epitope of $P$. berghei to primed CD8 ${ }^{+} \mathrm{T}$ cells [106].

To ensure full inhibition of blood stage malaria development $100 \%$ immunity is required. To achieve this, the very low number of hepatocytes that are actually infected has to be fully eliminated in the relatively short hepatic period, meaning that there is a need for a very large threshold of memory $\mathrm{T}$ cells to ensure immunity after epitope-specific immunization [106].

In short, the immune response in the liver is not fully elucidated yet and knowledge appears to be lacking. Plasmodium spp. likely exploit the immunotolerance of the liver to increase the relatively small number of sporozoites to a much larger number of merozoites. The liver stage therefore forms an ideal target for vaccine development. If successful, a vaccine would be able to stop the infection before any symptoms occur and would eliminate the spread of malaria, thus also contributing to its elimination.

While the liver offers a immunotolerogenic environment for the maturation of species of Ascaris, Schistosoma and Plasmodium, it should be noted that the liver has many other attributes that make it an attractive residence for these parasites. The liver sinusoids comprise a network of capillaries containing nutrient-rich blood from the intestine. The low blood pressure in the sinuses may offer an environment that supports growth, maturation and/or multiplication. Furthermore, the liver has a unique ability to regenerate and remodel itself, an attribute which could be exploited by the parasites to limit the deleterious effects of infection and inflammation and preserve their host tissue. The propensity of the liver to induce tolerance of foreign antigens, rather than immunity, is another attribute that may attract parasites, as well as other microorganisms, to this organ.

\section{Conclusions}

Immunologically the liver is a special organ where immune activation is reduced. This forms the ideal environment for species of Ascaris, Schistosoma and Plasmodium to mature to their next life stages, and multiply as is the case for Plasmodium spp. only. When migrating to the liver, the parasites are able evade the immune system. However, this does not always go according to plan. In the case of both Schistosoma spp. and Ascaris spp., the liver has been identified as a site of larval attrition. Understanding the molecular mechanisms behind this attrition could lead to the development of novel therapies. As for Plasmodium spp., the liver is a true bottleneck. It is at this life stage that the parasites multiply rapidly, before being released in the blood and spreading all over the host. The liver stage is therefore the ideal vaccine/drug target, as the parasite is still in relatively low numbers and concentrated in one organ. In short, the liver stage is understudied and more research is necessary to fully understand the molecular mechanisms and immune responses activated during parasitic invasion.

\section{Abbreviations}

LSECs: liver sinusoidal endothelial cells; APC: antigen-presenting cells; BAL: bronchoalveolar fluids; Treg: regulatory T cells; DC: dendritic cells; KC: Kupffer cells; TLR: Toll-Like receptor; MHC: major histocompatibility complex; HSC: hepatic stellate cells; MDSC: myeloid-derived suppressor cells; ROS: reactive oxygen species; CSP: circumsporozoite protein; TRAP: thrombospondin-related anonymous protein; HSPGs: human heparin sulphate proteoglycans; SPECT2: sporozoite microneme protein essential for cell traversal 2; PV: parasitophorous vacuole; MDA5: melanoma differentiation-associated gene 5 protein; MAVS: mitochondrial antiviral signalling; Irf: interferon-regulatory factors.

\section{Acknowledgements}

We thank two anonymous reviewers for their positive and constructive comments that have improved our manuscript. 


\section{Authors' contributions}

GD and CVH conceived, designed, edited and performed final revisions on the manuscript. GD, CVH and DGD wrote and edited the manuscript. GD, CVH and DGD read and approved the final manuscript. JC was involved in conceiving the concept of the manuscript. All authors read and approved the final manuscript.

\section{Funding}

This work was supported by the Irish Research Council Postgraduate Scholarship (Grant ID: GOIPG/2016/551) \& the Frank Jeal Scholarship, both awarded to GD.

\section{Availability of data and materials}

Not applicable.

\section{Ethics approval and consent to participate}

Not applicable.

\section{Consent for publication}

Not applicable.

\section{Competing interests}

The authors declare that they have no competing interests.

\section{Author details}

${ }^{1}$ Department of Zoology, School of Natural Sciences, Trinity College Dublin, Dublin 2, Ireland. ${ }^{2}$ School of Medicine, Trinity College Dublin, Dublin 2, Ireland.

${ }^{3}$ Department of Biology, Maynooth University, Maynooth, Co. Kildare, Ireland.

Received: 16 May 2019 Accepted: 4 November 2019

Published online: 08 November 2019

\section{References}

1. Read AF, Skorping A. The evolution of tissue migration by parasitic nematode larvae. Parasitology. 1995;111:359-71.

2. Mulcahy G, O’Neill S, Fanning J, McCarthy E, Sekiya M. Tissue migration by parasitic helminths - an immunoevasive strategy? Trends Parasitol. 2005;21:273-7.

3. Doherty DG. Immunity, tolerance and autoimmunity in the liver: a comprehensive review. J Autoimmun. 2016:66:60-75.

4. Calne RY. Immunological tolerance-the liver effect. Immunol Rev. 2000;174:280-2.

5. Devlin J, Doherty D, Thomson L, Wong T, Donaldson P, Portmann B, et al. Defining the outcome of immunosuppression withdrawal after liver transplantation. Hepatology. 1998;27:926-33.

6. Brandl K, Kumar V, Eckmann L. Gut-liver axis at the frontier of host-microbial interactions. Am J Physiol Gastrointest Liver Physiol. 2017;312:G413-9.

7. Doherty DG, O'Farrelly C. Innate and adaptive lymphoid cells in the human liver. Immunol Rev. 2000;174:5-20.

8. Thomson AW, Knolle PA. Antigen-presenting cell function in the tolerogenic liver environment. Nat Rev Immunol. 2010;10:753-66.

9. Crispe IN. Liver antigen-presenting cells. J Hepatol. 2011;54:357-65.

10. Guermonprez P, Valladeau J, Zitvogel L, Théry C, Amigorena S. Antigen presentation and T cell stimulation by dendritic cells. Annu Rev Immunol. 2002:20:621-67.

11. Tiegs G, Lohse AW. Immune tolerance: what is unique about the liver. J Autoimmun. 2010:34:1-6.

12. Crispe IN. Immune tolerance in liver disease. Hepatology. 2014:60:2109-17.

13. Steinman RM, Hawiger D, Nussenzweig MC. Tolerogenic dendritic cells. Annu Rev Immunol. 2003;21:685-711.

14. Kinoshita M, Uchida T, Sato A, Nakashima M, Nakashima H, Shono S, et al. Characterization of two F4/80-positive Kupffer cell subsets by their function and phenotype in mice. J Hepatol. 2010;53:903-10.

15. Davies LC, Jenkins SJ, Allen JE, Taylor PR. Tissue-resident macrophages. Nat Immunol. 2013:14:986-95.
16. Knolle P Schlaak J, Uhrig A, Kempf P, Meyer zum Büschenfelde KH, Gerken G. Human Kupffer cells secrete IL-10 in response to lipopolysaccharide (LPS) challenge. J Hepatol. 1995:22:226-9.

17. Wiegard C, Frenzel C, Herkel J, Kallen KJ, Schmitt E, Lohse AW. Murine liver antigen presenting cells control suppressor activity of CD4+CD25+ regulatory T cells. Hepatology. 2005;42:193-9.

18. Breous E, Somanathan S, Vandenberghe LH, Wilson JM. Hepatic regulatory $T$ cells and Kupffer cells are crucial mediators of systemic T cell tolerance to antigens targeting murine liver. Hepatology. 2009;50:612-21.

19. Limmer A, Ohl J, Kurts C, Ljunggren HG, Reiss Y, Groettrup M, et al. Efficient presentation of exogenous antigen by liver endothelial cells to CD8+ T cells results in antigen-specific T-cell tolerance. Nat Med. 2000;6:1348-54.

20. Lohse AW, Knolle PA, Bilo K, Uhrig A, Waldmann C, Ibe M, et al. Antigenpresenting function and B7 expression of murine sinusoidal endothelial cells and Kupffer cells. Gastroenterology. 1996;110:1175-81.

21. von Oppen N, Schurich A, Hegenbarth S, Stabenow D, Tolba R, Weiskirchen $\mathrm{R}$, et al. Systemic antigen cross-presented by liver sinusoidal endothelial cells induces liver-specific CD8 T-cell retention and tolerization. Hepatology. 2009:49:1664-72.

22. Kruse N, Neumann K, Schrage A, Derkow K, Schott E, Erben U, et al. Priming of CD4+ T cells by liver sinusoidal endothelial cells induces CD25low forkhead box protein 3- regulatory $T$ cells suppressing autoimmune hepatitis. Hepatology. 2009;50:1904-13.

23. Winau F, Hegasy G, Weiskirchen R, Weber S, Cassan C, Sieling PA, et al. Ito cells are liver-resident antigen-presenting cells for activating $T$ cell responses. Immunity. 2007:26:117-29.

24. Jiang G, Yang HR, Wang L, Wildey GM, Fung J, Qian S, et al. Hepatic stellate cells preferentially expand allogeneic CD4+ CD25+ FoxP3+ regulatory $T$ cells in an IL-2-dependent manner. Transplantation. 2008:86:1492-502.

25. Yang HR, Chou HS, Gu X, Wang L, Brown KE, Fung JJ, et al. Mechanistic insights into immunomodulation by hepatic stellate cells in mice: a critical role of interferon-gamma signaling. Hepatology. 2009;50:1981-91.

26. Höchst B, Schildberg FA, Sauerborn P, Gäbel YA, Gevensleben H, Goltz $D$, et al. Activated human hepatic stellate cells induce myeloid derived suppressor cells from peripheral blood monocytes in a CD44-dependent fashion. J Hepatol. 2013:59:528-35.

27. Bertolino P, Trescol-Biémont MC, Rabourdin-Combe C. Hepatocytes induce functional activation of naive CD8+T lymphocytes but fail to promote survival. Eur J Immunol. 1998;28:221-36.

28. Chen M, Tabaczewski P, Truscott SM, Van Kaer L, Stroynowski I. Hepatocytes express abundant surface class I MHC and efficiently use transporter associated with antigen processing, tapasin, and low molecular weight polypeptide proteasome subunit components of antigen processing and presentation pathway. J Immunol. 2005;175:1047-55.

29. Murrell KD, Eriksen L, Nansen P, Slotved HC, Rasmussen T. Ascaris suum: a revision of its early migratory path and implications for human ascariasis. J Parasitol. 1997:83:255-60.

30. McManus DP, Dunne DW, Sacko M, Utzinger J, Vennervald BJ, Zhou XN. Schistosomiasis. Nat Rev Dis Primers. 2018:4:13.

31. Ashley EA, Pyae Phyo A, Woodrow CJ. Malaria. Lancet. 2018;391:1608-21.

32. Roepstorff A, Eriksen L, Slotved HC, Nansen P. Experimental Ascaris suum infection in the pig: worm population kinetics following single inoculations with three doses of infective eggs. Parasitology. 1997;115:443-52.

33. Cowman AF, Healer J, Marapana D, Marsh K. Malaria: biology and disease Cell. 2016:167:610-24.

34. Pullan RL, Smith JL, Jasrasaria R, Brooker SJ. Global numbers of infection and disease burden of soil transmitted helminth infections in 2010 Parasit Vectors. 2014;7:37.

35. O'Lorcain $\mathrm{P}$, Holland CV. The public health importance of Ascaris lumbricoides. Parasitology. 2000;121(Suppl):S51-71.

36. Deslyper G, Holland CV. Overview on ascariasis in humans in South Asia. In: Singh SK, editor. Neglected tropical diseases-South Asia. Cham: Springer; 2017. p. 83-120.

37. Croll NA, Ghadirian E. Wormy persons: contributions to the nature and patterns of overdispersion with Ascaris lumbricoides, Ancylosotma duodenale, Necator americanus and Trichuris trichiura. Trop Geogr Med. $1981 ; 33: 241-8$ 
38. Holland CV, Asaolu SO, Crompton DW, Stoddart RC, Macdonald R, Torimiro SE. The epidemiology of Ascaris lumbricoides and other soiltransmitted helminths in primary school children from lle-lfe. Nigeria. Parasitology. 1989;99:275-85.

39. Holland CV. Predisposition to ascariasis: patterns, mechanisms and implications. Parasitology. 2009;136:1537-47.

40. Javid G, Wani NA, Gulzar GM, Khan BA, Shah AH, Shah OJ, et al. Ascarisinduced liver abscess. World I Surg. 1999;23:1191-4.

41. White EG. Chronic focal interstitial hepatitis in the pig. Vet J. 1941;97:155-72.

42. Stewart TB, Bidner TD, Southern LL, Simmons LA. Efficacy of fenbendazole against migrating Ascaris suum larvae in pigs. Am J Vet Res. 1984:45:984-6.

43. Hendricks MK, Moore SW, Millar AJ. Epidemiological aspects of liver abscesses in children in the Western Cape Province of South Africa. J Trop Pediatr. 1997:43:103-5.

44. Jungersen G, Fagerholm HP, Nansen P, Eriksen L. Development of patent Ascaris suum infections in pigs following intravenous administration of larvae hatched in vitro. Parasitology. 1999;1 19:503-8.

45. Masure D, Wang T, Vlaminck J, Claerhoudt S, Chiers K, Van den Broeck W, et al. The intestinal expulsion of the roundworm Ascaris suum is associated with eosinophils, intra-epithelial T cells and decreased intestinal transit time. PLoS Negl Trop Dis. 2013;7:e2588.

46. Masure $D$. The intestinal immune response against the porcine nematode Ascaris suum. Ghent: University Ghent; 2013.

47. Mitchell GF, Hogarth-Scott RS, Lewers HM, Edwards RD, Cousins G, Moore T. Studies on immune response to parasite antigens in mice. I. Ascaris suum larvae numbers and anti phosphorylcholine responses in infected mice of various strains and hypothymic nu/nu mice. Int Arch Allergy Immunol. 1976;56:64-78.

48. Lewis R, Behnke JM, Stafford P, Holland CV. The development of a mouse model to explore resistance and susceptibility to early Ascaris suum infection. Parasitology. 2006;132:289-300.

49. Dold C, Cassidy JP, Stafford P, Behnke JM, Holland CV. Genetic influence on the kinetics and associated pathology of the early stage (intestinal-hepatic) migration of Ascaris suum in mice. Parasitology. 2010;137:173-85.

50. Lewis R, Behnke JM, Cassidy JP, Stafford P, Murray N, Holland CV. The migration of Ascaris suum larvae, and the associated pulmonary inflammatory response in susceptible C57BL/6j and resistant CBA/Ca mice. Parasitology. 2007;134:1301-14.

51. Deslyper G, Colgan TJ, Cooper AJ, Holland CV, Carolan JC. A proteomic investigation of hepatic resistance to Ascaris in a murine model. PLoS Negl Trop Dis. 2016;10:e0004837.

52. Deslyper G, Holland CV, Colgan TJ, Carolan JC. The liver proteome in a mouse model for Ascaris suum resistance and susceptibility: evidence for an altered innate immune response. Parasit Vectors. 2019;12:402.

53. Nogueira DS, Gazzinelli-Guimarães PH, Barbosa FS, Resende NM, Silva CC, de Oliveira LM, et al. Multiple exposures to Ascaris suum induce tissue injury and mixed Th2/Th17 immune response in mice. PLoS Negl Trop Dis. 2016;10:e0004382.

54. Holland CV, Behnke JM, Dold C. Larval Ascariasis: impact, significance, and model organisms. In: Holland C, editor. Ascaris: the neglected parasite. Amsterdam: Elsevier; 2013. p. 107-25.

55. Colley DG, Bustinduy AL, Secor WE, King CH. Human schistosomiasis. Lancet. 2014;383:2253-64.

56. McManus DP, Loukas A. Current status of vaccines for schistosomiasis. Clin Microbiol Rev. 2008;21:225-42.

57. Olveda DU, Inobaya MT, McManus DP, Olveda RM, Vinluan ML, Ng SK, et al. Biennial versus annual treatment for schistosomiasis and its impact on liver morbidity. Int J Infect Dis. 2017;54:145-9.

58. Burke ML, Jones MK, Gobert GN, Li YS, Ellis MK, McManus DP. Immunopathogenesis of human schistosomiasis. Parasite Immunol. 2009:31:163-76.

59. Warren KS, Mahmoud AA, Cummings P, Murphy DJ, Houser HB. Schistosomiasis mansoni in Yemeni in California: duration of infection, presence of disease, therapeutic management. Am J Trop Med Hyg. 1974:23:902-9.

60. Barbosa MA, Pellegrino J, Coelho PM, Sampaio IB. Quantitative aspects of the migration and evolutive asynchronism of Schistosoma mansoni in mice. Rev Inst Med Trop Sao Paulo. 1978;20:121-32.
61. Rocha MO, Coelho PM. The importance of skin and pulmonary phases to the development of Schistosoma mansoni in albino mice. Rev Inst Med Trop Sao Paulo. 1980;22:157-63.

62. Jeremias WJ, Araújo FMG, Queiroz FR, Pais FSM, Mattos ACA, Salim ACM, et al. Comparative sequence analysis reveals regulation of genes in developing schistosomula of Schistosoma mansoni exposed to host portal serum. PLoS One. 2017;12:e0178829.

63. Shaker YM, Wu CH, el-Shobaki FA, Ashour E, Khattab HM, Draz HM, et al. Human portal serum stimulates cell proliferation in immature Schistosoma mansoni. Parasitology. 1998;117:293-9.

64. Draz HM, Ashour E, Shaker YM, Khattab HM, Wu CH, Wu GY. Host susceptibility to schistosomes: effect of host sera on cell proliferation of Schistosoma mansoni schistosomula. J Parasitol. 2008;94:1249-52.

65. Lu Z, Sessler F, Holroyd N, Hahnel S, Quack T, Berriman M, et al. Schistosome sex matters: a deep view into gonad-specific and pairingdependent transcriptomes reveals a complex gender interplay. Sci Rep. 2016;6:31150

66. Steinauer ML. The sex lives of parasites: investigating the mating system and mechanisms of sexual selection of the human pathogen Schistosoma mansoni. Int J Parasitol. 2009:39:1157-63.

67. Georgi JR, Dean DA, Chandiwanna SK. Quantification of Schistosoma mansoni in mouse lungs by radioassay and autoradiography of $75 \mathrm{Se}-$ labeled schistosomula. J Parasitol. 1982;68:1092-5.

68. Georgi JR, Dean DA, Mangold BL. Schistosoma mansoni: temporal distribution of radioselenium-labelled schistosomula in lungs of mice during the first two weeks of infection. Parasitology. 1983;86:31-6.

69. Mangold BL, Dean DA. Autoradiographic analysis of Schistosoma mansoni migration from skin to lungs in naive mice. Evidence that most attrition occurs after the skin phase. Am J Trop Med Hyg. 1983;32:785-9.

70. Wilson RA, Coulson PS, Dixon B. Migration of the schistosomula of Schistosoma mansoni in mice vaccinated with radiation-attenuated cercariae, and normal mice: an attempt to identify the timing and site of parasite death. Parasitology. 1986;92:101-16.

71. Mangold BL, Dean DA. The migration and survival of gamma-irradiated Schistosoma mansoni larvae and the duration of host-parasite contact in relation to the induction of resistance in mice. Parasitology. 1984;88:249-65.

72. Mastin AJ, Bickle QD, Wilson RA. Schistosoma mansoni: migration and attrition of irradiated and challenge schistosomula in the mouse. Parasitology. 1983:87:87-102.

73. Von Lichtenberg F, Correa-Oliveira R, Sher A. The fate of challenge schistosomula in the murine anti-schistosome vaccine model. Am 」 Trop Med Hyg. 1985;34:96-106.

74. Wilson RA. The saga of schistosome migration and attrition. Parasitology. 2009;136:1581-92.

75. Georgi JR, Wade SE, Dean DA. Attrition and temporal distribution of Schistosoma mansoni and S. haematobium schistosomula in laboratory mice. Parasitology. 1986;93:55-70.

76. Imbert-Establet D, Vera C, Sellin B, Jourdane J. The mouse as a suitable host for an isolate of Schistosoma haematobium from Niger. J Helminthol. 1992;66:1-5.

77. Laxer MJ, Tuazon CU. Migration of 75Se-methionine-labelled Schistosoma japonicum in normal and immunized mice. J Infect Dis. 1992;166:1133-8.

78. WHO. World malaria report 2016. Geneva: World Health Organization; 2016.

79. van Wolfswinkel ME, Langenberg MCC, Wammes $\amalg$, Sauerwein RW, Koelewijn R, Hermsen CC, et al. Changes in total and differential leukocyte counts during the clinically silent liver phase in a controlled human malaria infection in malaria-naïve Dutch volunteers. Malar J. 2017;16:457.

80. Mo AX, McGugan G. Understanding the liver-stage biology of malaria parasites: insights to enable and accelerate the development of a highly efficacious vaccine. Am J Trop Med Hyg. 2018:99:827-32.

81. Ejigiri I, Sinnis P. Plasmodium sporozoite-host interactions from the dermis to the hepatocyte. Curr Opin Microbiol. 2009:12:401-7.

82. Frevert U, Sinnis P, Cerami C, Shreffler W, Takacs B, Nussenzweig V. Malaria circumsporozoite protein binds to heparan sulfate proteoglycans associated with the surface membrane of hepatocytes. J Exp Med. 1993;177:1287-98. 
83. Sinnis P, Willnow TE, Briones MR, Herz J, Nussenzweig V. Remnant lipoproteins inhibit malaria sporozoite invasion of hepatocytes. J Exp Med. 1996;184:945-54.

84. Pradel G, Frevert U. Malaria sporozoites actively enter and pass through rat Kupffer cells prior to hepatocyte invasion. Hepatology. 2001;33:1154-65.

85. Frevert U, Engelmann S, Zougbédé S, Stange J, Ng B, Matuschewski K, et al. Intravital observation of Plasmodium berghei sporozoite infection of the liver. PLoS Biol. 2005;3:e192.

86. Tavares J, Formaglio P, Thiberge S, Mordelet E, Van Rooijen N, Medvinsky A, et al. Role of host cell traversal by the malaria sporozoite during liver infection. J Exp Med. 2013;210:905-15.

87. Usynin I, Klotz C, Frevert U. Malaria circumsporozoite protein inhibits the respiratory burst in Kupffer cells. Cell Microbiol. 2007;9:2610-28.

88. Ishino T, Chinzei Y, Yuda M. A Plasmodium sporozoite protein with a membrane attack complex domain is required for breaching the liver sinusoidal cell layer prior to hepatocyte infection. Cell Microbiol. 2005:7:199-208

89. Mota MM, Pradel G, Vanderberg JP, Hafalla JC, Frevert U, Nussenzweig RS, et al. Migration of Plasmodium sporozoites through cells before infection. Science. 2001;291:141-4.

90. Carrolo M, Giordano S, Cabrita-Santos L, Corso S, Vigário AM, Silva S, et al. Hepatocyte growth factor and its receptor are required for malaria infection. Nat Med. 2003;9:1363-9.

91. Coppi A, Tewari R, Bishop JR, Bennett BL, Lawrence R, Esko JD, et al. Heparan sulfate proteoglycans provide a signal to Plasmodium sporozoites to stop migrating and productively invade host cells. Cell Host Microbe. 2007;2:316-27.

92. Risco-Castillo V, Topçu S, Marinach C, Manzoni G, Bigorgne AE, Briquet $S$, et al. Malaria sporozoites traverse host cells within transient vacuoles. Cell Host Microbe. 2015;18:593-603.

93. Sturm A, Amino R, van de Sand C, Regen T, Retzlaff S, Rennenberg A, et al. Manipulation of host hepatocytes by the malaria parasite for delivery into liver sinusoids. Science. 2006;313:1287-90.

94. Graewe S, Rankin KE, Lehmann C, Deschermeier C, Hecht L, Froehlke $U$, et al. Hostile takeover by Plasmodium: reorganization of parasite and host cell membranes during liver stage egress. PLoS Pathog. 2011;7:e1002224.

95. Richter J, Franken G, Mehlhorn H, Labisch A, Häussinger D. What is the evidence for the existence of Plasmodium ovale hypnozoites? Parasito Res. 2010;107:1285-90.
96. Groger M, Fischer HS, Veletzky L, Lalremruata A, Ramharter M. A systematic review of the clinical presentation, treatment and relapse characteristics of human Plasmodium ovale malaria. Malar J. 2017;16:112.

97. Adams JH, Mueller I. The biology of Plasmodium vivax. Cold Spring Harb Perspect Med. 2017;7:a025585.

98. White MT, Shirreff G, Karl S, Ghani AC, Mueller I. Variation in relapse frequency and the transmission potential of Plasmodium vivax malaria. Proc Biol Sci. 2016;283:20160048.

99. Battle KE, Karhunen MS, Bhatt S, Gething PW, Howes RE, Golding N, et al. Geographical variation in Plasmodium vivax relapse. Malar J. 2014;13:144.

100. Betuela I, Rosanas-Urgell A, Kiniboro B, Stanisic DI, Samol L, de Lazzari

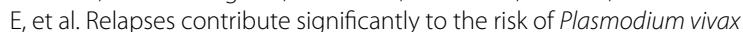
infection and disease in Papua New Guinean children 1-5 years of age. J Infect Dis. 2012;206:1771-80.

101. Robinson LJ, Wampfler R, Betuela I, Karl S, White MT, Li Wai Suen CS, et al. Strategies for understanding and reducing the Plasmodium vivax and Plasmodium ovale hypnozoite reservoir in Papua New Guinean children: a randomised placebo-controlled trial and mathematical model. PLoS Med. 2015;12:e1001891.

102. Markus MB. New evidence for hypnozoite-independent Plasmodium vivax malarial recurrences. Trends Parasitol. 2018;34:1015-6.

103. Gowda DC, Wu X. Parasite recognition and signalling mechanisms in innate immune responses to malaria. Front Immunol. 2018;9:3006.

104. Kurtovic L, Behet MC, Feng G, Reiling L, Chelimo K, Dent AE, et al. Human antibodies activate complement against Plasmodium falciparum sporozoites, and are associated with protection against malaria in children. BMC Med. 2018;16:61.

105. Weiss WR, Sedegah M, Beaudoin RL, Miller LH, Good MF. CD8+ T cells (cytotoxic/suppressors) are required for protection in mice immunized with malaria sporozoites. Proc Natl Acad Sci USA. 1988;85:573-6.

106. Silvie $O$, Amino R, Hafalla JC. Tissue-specific cellular immune responses to malaria pre-erythrocytic stages. Curr Opin Microbiol. 2017:40:160-7.

107. Chakravarty S, Cockburn IA, Kuk S, Overstreet MG, Sacci JB, Zavala F. CD8+ T lymphocytes protective against malaria liver stages are primed in skin-draining lymph nodes. Nat Med. 2007;13:1035-41.

\section{Publisher's Note}

Springer Nature remains neutral with regard to jurisdictional claims in published maps and institutional affiliations.
Ready to submit your research? Choose BMC and benefit from:

- fast, convenient online submission

- thorough peer review by experienced researchers in your field

- rapid publication on acceptance

- support for research data, including large and complex data types

- gold Open Access which fosters wider collaboration and increased citations

- maximum visibility for your research: over $100 \mathrm{M}$ website views per year

At BMC, research is always in progress.

Learn more biomedcentral.com/submissions 